\title{
DHA Metabolism: Targeting the Brain and Lipoxygenation
}

\author{
M. Picq • P. Chen • M. Perez • M. Michaud • E. Véricel • \\ M. Guichardant $\cdot$ M. Lagarde
}

Received: 5 April 2010 /Accepted: 5 April 2010 /Published online: 28 April 2010

(C) The Author(s) 2010. This article is published with open access at Springerlink.com

\begin{abstract}
Docosahexaenoic acid (DHA), the end-product of the metabolism of omega-3 family fatty acids, is the main polyunsaturated fatty acid of the brain, but its accumulation is incompletely understood. This paper reviews how it could accumulate through specific uptake of DHA-containing lysophosphatidylcholine (LysoPCDHA). DHA migrates very easily from the $s n-2$ position of LysoPC, which could be considered as the physiological form of polyunsaturated LysoPC, to the $s n-1$ position, which is much more stable. An approach preventing migration by acetylating the $s n-1$ position, while retaining the main physico-chemical properties of the carrier, is described. Also, the double lipoxygenation and bond-isomerization of DHA into $10(S), 17(S)$-docosahexa-4Z,7Z,11E,13Z,15E,19Zenoic acid, named PDX, by soybean lipoxygenase is described. As in other E,Z,E conjugated trienes, PDX is shown to inhibit human blood platelet aggregation at submicromolar concentrations.
\end{abstract}

Keywords DHA · Lysophosphatidylcholine · PDX

\footnotetext{
M. Picq $\cdot$ P. Chen $\cdot$ M. Perez $\cdot$ M. Michaud $\cdot$ E. Véricel $\cdot$

M. Guichardant $\cdot$ M. Lagarde

Université de Lyon, UMR 870 Inserm/Insa-Lyon,

Univ-Lyon 1, Inra,

1235 Lyon, France

M. Lagarde $(\square)$

UMR 870/RMND, IMBL,

Bldg Louis Pasteur, Insa-Lyon,

69621 Villeurbanne, France

e-mail: Michel.Lagarde@insa-lyon.fr
}

\section{Introduction}

Docosahexaenoic acid (DHA, 22:6w3) is a long-chain fatty acid of the omega-3 family derived from the essential precursor linolenic acid (LNA, 18:3w3). DHA is the main end-product of LNA after successive desaturations and elongations, a metabolic cascade that is assumed to be weak in humans $[1,2]$.

DHA is mainly esterified in membrane phospholipids of the brain, retina, and spermatozoa [3]. Its function in the two former structures has been well documented, and it is assumed to play an important role in brain development, learning ability, and visual acuity. One outstanding issue, however, remains unresolved, namely to understand how DHA specifically accumulates in these organs, especially in brain which is tightly separated from the blood circulation by the blood-brain barrier.

Furthermore, DHA, being highly unsaturated, has the potential to be oxygenated by various lipoxygenases to produce diverse oxylipins. A pioneer work has shown that DHA can be converted by the so-called 5-lipoxygenase (responsible for leukotriene production from arachidonic acid) into 7-hydroperoxy-DHA and its 7-hydroxy derivative 7-HDoHE [4]. A further report has described the formation of both 11- and 14-hydroperoxy-DHA and their hydroxy derivatives 11- and 14-HDoHE by platelet lipoxygenase [5]. More recently, a series of di- and tri-hydroxy derivatives of DHA have been described as conjugated trienes and tetraenes derived from double or triple oxidation. The most bioactive of these have been termed resolvins because they promote the resolution of inflammation [6] and protectin D1 (PD1) or neuroprotectin D1 (NPD1) because of a potent anti-inflammatory potential [7] and potent neuroprotective effect [8], respectively. The anti-inflammatory effects of these compounds could 
beneficially affect the function of neurological tissues including brain.

The present paper reports on the targeting of the brain with DHA-containing phospholipids and on the oxygenation of DHA by an omega-6 lipoxygenase, forming an isomer of PD1 that we have named PDX.

\section{Targeting the Brain with Choline Phospholipids}

It is generally assumed that the transport of fatty acids to the brain occurs from the non-esterified pool (NEFA) bound to serum albumin. NEFA would then cross the blood-brain barrier (BBB) as a result of competition between the hydrophobic domain of albumin and that of the endothelial layer of the BBB, as reported for other organs [9]. However, serum albumin also binds lysophospholipids, mainly lysophosphatidylcholine (LysoPC), also called lysolecithin. It has been known for years that LysoPC is produced by lecithin cholesterol acyl transferase as a result of transferring, within high-density lipoproteins (HDL), the acyl chain from the $s n-2$ position of HDL-PC to free cholesterol, producing cholesterylester and LysoPC; the latter is further transferred to albumin [10]. The resulting LysoPC has a saturated acyl chain at the $s n-1$ position. However, the release of unsaturated LysoPC resulting from the cleavage of PC by the liver phospholipase A1 could not be excluded, so we have considered the possibility that unsaturated LysoPC, including DHAcontaining LysoPC (LysoPC-DHA), can become associated with albumin and transfer across the BBB as do NEFA.

First, a series of ${ }^{14} \mathrm{C}$-labeled FA (arachidonic, linoleic, oleic, and palmitic acids), either as NEFA or esterified at the $s n-2$ position of LysoPC, were bound to albumin and injected into the rat to look at the brain accretion of each FA. The three unsaturated FA, but not palmitic acid, were taken up by the brain more efficiently (from 5 - to 10 -fold) when in LysoPC compared to the NEFA form [11].

Secondly, DHA in both forms was injected to another set of rats, and its incorporation into the brain, heart, kidney, and liver was measured. Again, DHA uptake by the brain was around 10-fold more efficient when injected as LysoPC-DHA compared to DHA. This was specific for the brain, since there was no preference between the accumulation of the two forms of DHA in the kidney, and the heart and liver even preferred DHA to Lyso PC-DHA [12].

This approach clearly showed that LysoPC-DHA is a privileged form of transport of DHA to the brain.

In another approach, ${ }^{13} \mathrm{C}$-labeled DHA esterified in triacylglycerols (TG), the form of DHA in fish oil, was ingested by rats, and the ${ }^{13} \mathrm{C}$-DHA was followed in various blood compartments and brain phospholipids by gas- chromatography combustion isotope ratio mass spectrometry. Focusing on the serum albumin pool, it could be seen that DHA accumulated in LysoPC-DHA with only a slight decrease over time (evaluated for $72 \mathrm{~h}$ ), while nonesterified DHA transiently peaked with a return to basal by $12 \mathrm{~h}$ post-intake. In parallel, ${ }^{13} \mathrm{C}$-DHA was followed in brain phospholipids and was found to increase in the main glycerophospholipids/phosphatidylcholine (PC) and phosphatidylethanolamine, until $72 \mathrm{~h}$ post-intake. This fits with the hypothesis that LysoPC-DHA, rather than non-esterified DHA, is the main carrier of DHA transported by albumin [13].

The same approach was then used in humans with measurement of ${ }^{13} \mathrm{C}$-DHA accumulation in blood platelets and red cells, the former compartment being known to rapidly accumulate PUFA from the NEFA pool bound to albumin, whereas the latter compartment is accepted as an index of the brain DHA accretion.

The pattern for the kinetic accumulation of ${ }^{13} \mathrm{C}$-DHA in serum albumin was quite similar to that observed in rats, with a transient peak in the NEFA pool and return to basal by $12 \mathrm{~h}$ post-intake, and accumulation in LysoPC with a slow decrease over time.

The incorporation of DHA into platelet phospholipids was rapid and attained a plateau when ${ }^{13} \mathrm{C}$-DHA in the NEFA pool of albumin had returned to basal; a lag phase of $8 \mathrm{~h}$ followed by a constant rise until 3 days post-intake could be seen in red cells. This is in good agreement with LysoPC-DHA being the main source of DHA for red cells, whereas platelets take up DHA rather uniquely from the NEFA pool [14].

Finally, ${ }^{13} \mathrm{C}$-DHA esterified in $\mathrm{PC}$ was ingested by humans. The kinetics of ${ }^{13} \mathrm{C}$-DHA in serum albumin NEFA and LysoPC, as well in platelets and red cells, were not markedly different from those obtained after ingestion of ${ }^{13} \mathrm{C}$-DHA in triacylglycerols. However, we were able to detect LysoPC-DHA itself in red cells as additional evidence for considering LysoPC as a main source of DHA to red cells and in turn to the brain [15].

We then analyzed the content of human and rat plasma for LysoPC as well as their FA composition and position on the glycerol backbone. We found substantial amounts of PUFA in LysoPC, with linoleic, arachidonic, and docosahexaenoic acids being the main species; around $50 \%$ of the PUFA was in the $s n-2$ position of the glycerol moiety. Provided that three quarters of the DHA in the $s n-2$ position migrates to the $s n-1$ position within $20 \mathrm{~min}$ at $37^{\circ} \mathrm{C}$ and physiological $\mathrm{pH} 7.4$, this means that the main form of production of LysoPC-DHA is the one with DHA at the $s n$ 2 position [16].

In summary, we came to the following scheme in which, once absorbed at the intestinal stage, DHA may circulate in different forms, including PC (the main circulating glycer- 
ophospholipid), cholesterylesters, and TG that are rapidly hydrolyzed by lipoprotein lipase to provide non-esterified DHA. Part of PC-DHA could then be hydrolyzed by the socalled endothelial lipase, which has recently been described as selective for DHA-containing phospholipids, to release 1-lyso,2-DHA-glycerophosphocholine (LysoPC-DHA) [17]. Both LysoPC-DHA and non-esterified DHA are bound to albumin and could then provide DHA to the brain, with the former being more efficient. Figure 1 summarizes this scheme.

Considering that DHA is at the $s n-1$ position of LysoPC, the most stable form of LysoPC-DHA (see above) is not the physiological form to be reacylated in the brain, because DHA is stored in tissues at the $s n-2$ position; we therefore made an attempt to keep DHA at this position. We first blocked the alcohol function at the $s n-1$ position by acetylation. This could be achieved by chemical treatment of 1-lyso,2-DHA-glycerophosphocholine with acetic anhydride [18]. The product, 1-acetyl,2-DHA-glycerophosphocholine, has been named AceDoPC. More recently, we set up a one-step enzymatic way to generate AceDoPC from PC-DHA, which improved the yield of the isomer with DHA at the $s n-2$ position [19] (Fig. 2). AceDoPC is produced in order to assess its metabolism in humans (use of ${ }^{13} \mathrm{C}$-labeled AceDoPC) and to test its effects on different neurobiological disorders in rodents.

\section{Lipoxygenation of DHA into Dihydroxylated Conjugated Triene Derivatives}

PD1 has been shown by the group of Serhan to be produced by treatment of DHA by soybean lipoxygenase [20]. This lipoxygenase is an omega-6 lipoxygenase, which produces 17(S)-HOO-docosahexa-4Z,7Z,10Z,13Z,15E,19Z-enoic ac-

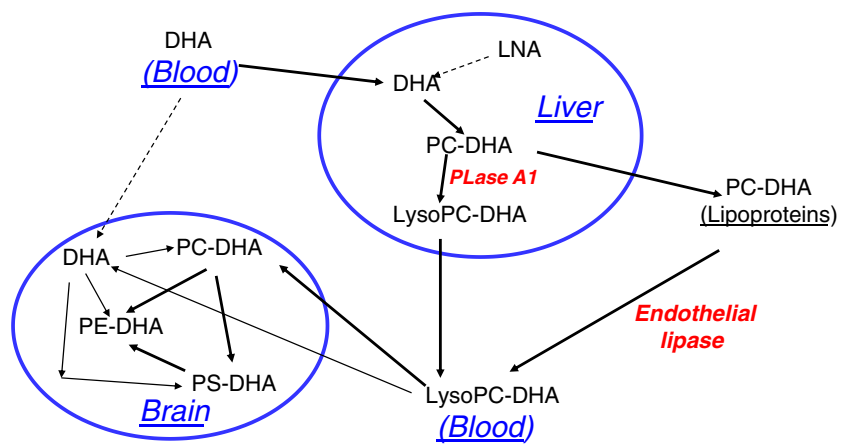

Fig. 1 Proposed metabolism for DHA with ultimate incorporation into brain phospholipids. DHA is weakly produced from linolenic acid $(L N A)$ in the liver. LysoPC-DHA may be produced from PC-DHA hydrolysis by liver phospholipase $\mathrm{A} 1$ and released and/or by endothelial lipase from circulating PC-DHA. LysoPC-DHA may be taken up by the brain to be reacylated into PC and/or hydrolyzed to provide DHA to be esterified into phospholipids within the brain. Interconversion between brain phospholipids may also occur

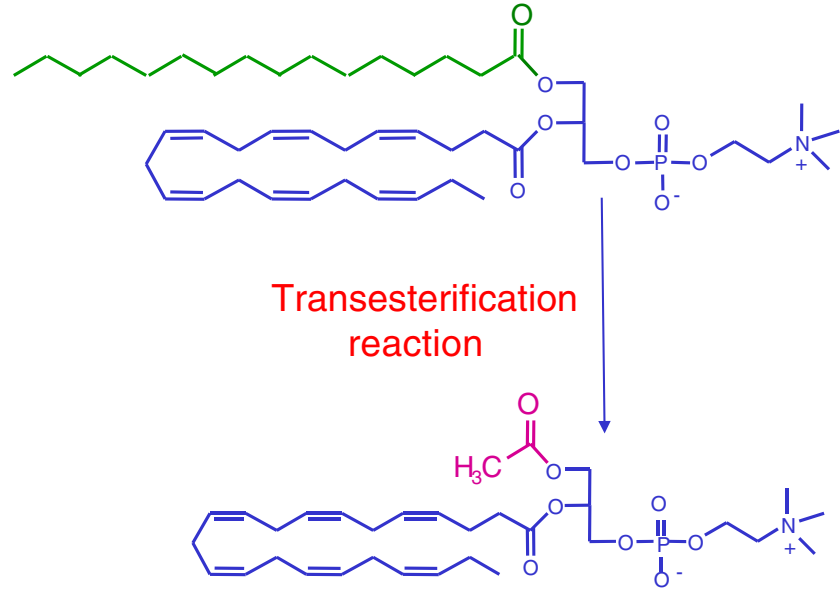

1-acetyl,2-docosahexaenoyl-glycerophosphocholine «AceDoPC»

Fig. 2 Scheme for the preparation of AceDoPC from PC-DHA. PCDHA purified from the micro-algae Crypthecodinium cohnii is submitted for transesterification by the action of an immobilized triglyceride lipase in the presence of ethylacetate. AceDoPC is finally purified by HPLC. ${ }^{13} \mathrm{C}$-Labeled DHA is prepared by cultivating the micro-algae in the presence of ${ }^{13} \mathrm{C}$-labeled acetate

id (17(S)-HpDoHE) from DHA, which is easily reduced into its hydroxy derivative 17(S)-HDoHE. PD1 has been further characterized as $10(S), 17(S)$-diOH-docosahexa$4 Z, 7 Z, 11 E, 13 E, 15 Z, 19 Z$-enoic acid, assuming the formation of an epoxide intermediate as is well known for leukotriene B4 formation from arachidonic acid [21].

In the mean time, Butovich et al. [22] described the formation of 10,17(S)-diOH-docosahexa-4Z,7Z,11E,13Z, $15 E, 19 Z$-enoic acid and called it PD1. However, the two structures differ in their conjugated triene cis/trans geometry, which is $E, Z, E$ in the latter molecule while it is $E, E, Z$ in PD1, according to Serhan.

We have reconsidered the conversion of DHA by soybean lipoxygenase type 1-B and found a major product as $17(S)$-HDoHE and a second one as $10(S), 17(S)$-diOH-

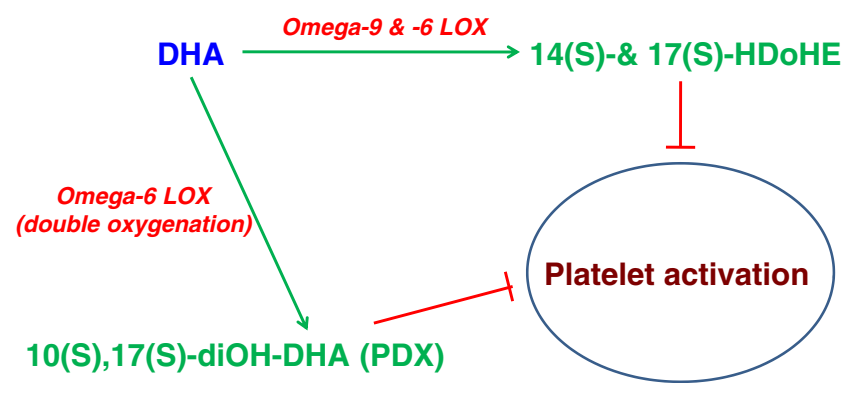

Fig. 3 Inhibition of platelet activation by lipoxygenase $(L O X)$ products of DHA. Platelet aggregation is inhibited by monoxydroxy derivative of DHA and by the dihydroxy derivative conjugated triene having the $E, Z, E$ geometry, issued from the double lipoxygenation of DHA, called PDX, an isomer of neuroprotectin D1 
docosahexa-4Z,7Z,11E,13Z,15E,19Z-enoic acid in agreement with Butovich, although this author did not determine the stereoconfiguration of carbon 10. Our results strongly suggest that the product we have characterized is likely to derive from a double lipoxygenation of DHA. We have named it PDX to differentiate it from PD1 [23].

Interestingly, PDX and other fatty acids having the $E, Z, E$ conjugated triene geometry are potent inhibitors of platelet aggregation, whereas those with the $E, E, Z$ geometry are not. This makes the $E, Z, E$ conjugated triene motif relevant in the inhibition of platelet aggregation by lipoxygenase products.

These data follow previous evidence showing that monohydroxy derivatives of DHA, produced by omega- 9 and omega-6 lipoxygenases, inhibit thromboxane-induced platelet aggregation [24], summarized in Fig. 3.

Altogether, our data show that DHA metabolites may attenuate platelet activation, in addition to DHA competition with the arachidonic acid cascade from endogenous phospholipids. It is conceivable that these metabolites may also reduce inflammatory processes in neural tissues including brain.

Acknowledgments This work is supported by INSERM, French Ministry of Research, ANR PNRA 2007, and LISA Carnot Institute.

Open Access This article is distributed under the terms of the Creative Commons Attribution Noncommercial License which permits any noncommercial use, distribution, and reproduction in any medium, provided the original author(s) and source are credited.

\section{References}

1. Burdge GC, Jones AE, Wootton SA (2002) Eicosapentaenoic and docosapentaenoic acids are the principal products of alphalinolenic acid metabolism in young men. Br J Nutr 88:355-363

2. Brenna JT, Salem N Jr, Sinclair AJ, Cunnane SC (2009) Alphalinolenic acid supplementation and conversion to n-3 long-chain polyunsaturated fatty acids in humans. Prostaglandins Leukot Essent Fatty Acids 80:85-91, Review

3. Salem N Jr, Litman B, Kim HY, Gawrisch K (2001) Mechanisms of action of docosahexaenoic acid in the nervous system. Lipids 3:945-959, Review

4. Lee TH, Mencia-Huerta JM, Shih C, Corey EJ, Lewis RA, Austen KF (1984) Effects of exogenous arachidonic, eicosapentaenoic, and docosahexaenoic acids on the generation of 5-lipoxygenase pathway products by ionophore-activated human neutrophils. J Clin Invest 74:1922-1933

5. Aveldano MI, Sprecher H (1983) Synthesis of hydroxy fatty acids from 4, 7, 10, 13, 16, 19-[1-14C] docosahexaenoic acid by human platelets. J Biol Chem 258:9339-9343

6. Hong S, Gronert K, Devchand PR, Moussignac RL, Serhan CN (2003) Novel docosatrienes and 17S-resolvins generated from docosahexaenoic acid in murine brain, human blood, and glial cells. Autacoids in anti-inflammation. J Biol Chem 278:1467714687

7. Bannenberg GL, Chiang N, Ariel A, Arita M, Tjonahen E, Gotlinger KH, Hong S, Serhan CN (2005) Molecular circuits of resolution: formation and actions of resolvins and protectins. J Immunol 174:5884-5894

8. Bazan NG, Marcheselli VL, Cole-Edwards K (2005) Brain response to injury and neurodegeneration: endogenous neuroprotective signaling. Ann N Y Acad Sci 1053:137-147, Review

9. Hamilton JA (2007) New insights into the roles of proteins and lipids in membrane transport of fatty acids. Prostaglandins Leukot Essent Fatty Acids 77(5-6):355-361

10. Subbaiah PV (1986) (1992) Lysolecithin acyltransferase of human plasma: assay and characterization of enzyme activity. Meth Enzymol 129:790-797

11. Thies F, Delachambre MC, Bentejac M, Lagarde M, Lecerf J (1992) Unsaturated fatty acids esterified in 2-acyl-1-lysophosphatidylcholine bound to albumin are more efficiently taken up by the young rat brain than the unesterified form. J Neurochem 59:1110-1116

12. Thies F, Pillon C, Moliere P, Lagarde M, Lecerf J (1994) Preferential incorporation of $s n-2$ lysoPC DHA over unesterified DHA in the young rat brain. Am J Physiol 267:R1273-R1279

13. Brossard N, Croset M, Lecerf J, Pachiaudi C, Normand S, Chirouze V, Macovschi O, Riou JP, Tayot JL, Lagarde M (1996) Metabolic fate of an oral tracer dose of [13C]docosahexaenoic acid triglycerides in the rat. Am J Physiol 270:R846-R854

14. Brossard N, Croset M, Normand S, Pousin J, Lecerf J, Laville M, Tayot JL, Lagarde M (1997) Human plasma albumin transports [13C]docosahexaenoic acid in two lipid forms to blood cells. J Lipid Res 7:1571-1582

15. Lemaitre-Delaunay D, Pachiaudi C, Laville M, Pousin J, Armstrong M, Lagarde M (1999) Blood compartmental metabolism of docosahexaenoic acid (DHA) in humans after ingestion of a single dose of [(13)C]DHA in phosphatidylcholine. J Lipid Res 40:1867-1874

16. Croset M, Brossard N, Polette A, Lagarde M (2000) Characterization of plasma unsaturated lysophosphatidylcholines in human and rat. Biochem J 345:61-67

17. Chen S, Subbaiah PV (2007) Phospholipid and fatty acid specificity of endothelial lipase: potential role of the enzyme in the delivery of docosahexaenoic acid (DHA) to tissues. Biochim Biophys Acta 1771:1319-1328

18. Polette A, Deshayes C, Chantegrel B, Croset M, Armstrong JM, Lagarde M (1999) Synthesis of acetyl, docosahexaenoylglycerophosphocholine and its characterization using nuclear magnetic resonance. Lipids 34:1333-1337

19. Lagarde M, Guichardant M, Picq M, Michaud S, Doutheau A (2008) Method for preparing acetyl,docosahexaenoyl-glycerophosphocholine and use thereof for the delivery of polyunsaturated fatty acids. WO/2008/068413.

20. Ariel A, Li PL, Wang W, Tang WX, Fredman G, Hong S, Gotlinger KH, Serhan CN (2005) The docosatriene protectin D1 is produced by $\mathrm{TH} 2$ skewing and promotes human $\mathrm{T}$ cell apoptosis via lipid raft clustering. J Biol Chem 280:43079-43086

21. Serhan CN, Gotlinger K, Hong S, Lu Y, Siegelman J, Baer T, Yang R, Colgan SP, Petasis NA (2006) Anti-inflammatory actions of neuroprotectin D1/protectin D1 and its natural stereoisomers: assignments of dihydroxy-containing docosatrienes. J Immunol 176:1848-1859

22. Butovich IA (2005) On the structure and synthesis of neuroprotectin D1, a novel anti-inflammatory compound of the docosahexaenoic acid family. J Lipid Res 46:2311-2314

23. Chen P, Fenet B, Michaud S, Tomczyk N, Véricel E, Lagarde M, Guichardant M (2009) Full characterization of PDX, a neuroprotectin/protectin D1 isomer, which inhibits blood platelet aggregation. FEBS Lett 583:3478-3484

24. Croset M, Sala A, Folco GC, Lagarde M (1988) Inhibition by lipoxygenase products of TXA2-like responses of platelets and vascular smooth muscle. 14-Hydroxy from $22: 6 n-3$ is more potent than 12-HETE. Biochem Pharmacol 37:1275-1280 